\title{
Successful use of spinal anesthesia for an urgent cesarean section in a parturient with a severe Klippel- Trénaunay syndrome
}

\author{
Gian Luigi Gonnella, Marco Scorzoni, Stefano Catarci, \\ Bruno Antonio Zanfini, and Gaetano Draisci \\ Department of Anesthesiology and Intensive Care Medicine, Catholic University School of Medicine, Rome, Italy
}

Klippel-Trénaunay syndrome (KTS) is a congenital malformation with an incidence of 1 out of 27,500 live births described in 1900 including a triad of port-wine stain/capillary vascular malformation, venous malformation/varicose veins, soft tissue, and bony hypertrophy in affected limbs [1]. Although a combined spinal-epidural technique for an elective cesarean section and an epidural analgesia for vaginal delivery have been already presented [2,3], this is the first report that describes spinal anesthesia for an urgent cesarean section in a patient with KTS.

A 23-year-old woman (gravidity: 1, parity: 0) was referred to our Anesthesia Preadmission Clinic at 37 weeks of gestation because of KTS complicating her pregnancy. She weighed 115 $\mathrm{kg}$ and was $170 \mathrm{~cm}$ in height (body mass index $39.8 \mathrm{~kg} / \mathrm{m}^{2}$ ). She had been diagnosed with KTS at the age of 16 and received several surgical treatments for her right foot varicosities and opioid therapy till the beginning of gestation because of pain in the right leg. She did not have a history of thrombosis or hemorrhage. On hospital admission at 37 weeks of gestation for planned delivery, physical examination showed prominent hypertrophy and multiple varicosities of the right leg (Fig. 1). The circumferences of the thigh, calf, ankle, and knee were 12, 15,

Corresponding author: Gian Luigi Gonnella, M.D.

Department of Anesthesiology and Intensive Care Medicine, Catholic University School of Medicine, Largo Agostino Gemelli, 8 Roma 00168, Italy

Tel: 39-0630154542, Fax: 39-063013450

Email: gianluigigonnella@yahoo.it

ORCID: https://orcid.org/0000-0002-7115-4408

Received: February 23, 2018.

Revised: March 20, 2018 (1st); March 27, 2018 (2nd).

Accepted: March 28, 2018.

Korean J Anesthesiol 2018 October 71(5): 411-412

https://doi.org/10.4097/kja.d.18.00050
11 , and $10 \mathrm{~cm}$, respectively, larger than the left side. Laboratory studies revealed a normal coagulation profile and hemoglobin $(\mathrm{Hb})$ count of $8.5 \mathrm{~g} / \mathrm{dl}$. She was scheduled for a magnetic resonance imaging (MRI) scan to determine the existence of arterio-venous malformations (AVM) or hemangiomas in the pelvis, birth canal, spinal cord, bronchial tube, and brain before delivery, but she had not yet undergone MRI. She was admitted to our delivery unit at 38 weeks of gestation for an urgent cesarean section due to abnormal cardiotocography (type 2 urgency according to Lucas' classification). On admission, she was anxious and had breakfast two hours before. On physical examination, her airway revealed a Mallampati Class III and her back had normal anatomy with clearly palpable landmarks with no evidence of port-wine stains. Ultrasound revealed no signs of detectable vascular abnormalities. She refused any attempt

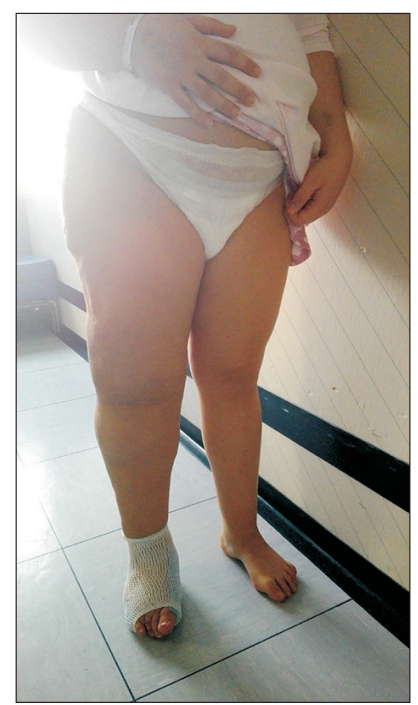

Fig. 1. Detail of the patient's affected leg.

(c) This is an open-access article distributed under the terms of the Creative Commons Attribution Non-Commercial License (http://creativecommons.org/ licenses/by-nc/4.0/), which permits unrestricted non-commercial use, distribution, and reproduction in any medium, provided the original work is properly cited. 
of awake intubation. MRI of lumbar spine performed six years ago showed no AVM or hemangioma. After discussing rapidly the risks and benefits of general and spinal anesthesia, we decided that spinal anesthesia was safer in this case. However, we informed her the possibility of intraoperative conversion to general anesthesia in case significant hemorrhage occurred during the operation. Two 16-gauge intravenous catheters were placed in the left antecubital fossa bilaterally. Aspiration and antibiotic prophylaxis were performed routinely. In the meantime, an intravenous bolus of $0.9 \%$ saline $500 \mathrm{ml}$ was administered before neuraxial anesthesia. Monitors included an electrocardiogram, finger pulse oximetry, and noninvasive blood pressure. In sitting position, spinal anesthesia at $\mathrm{L}_{3-4}$ intervertebral space was performed with $5 \mu \mathrm{g}$ of sufentanil and $10 \mathrm{mg}$ of hyperbaric bupivacaine. Then, the patient was positioned in the supine position with uterus displacement and her legs were lifted 30 degrees. A T4 anesthetic block was obtained without developing hypotension after neuraxial block. Her systolic blood pressure remained greater than $100 \mathrm{mmHg}$. A female baby weighing 2,964 $\mathrm{g}$ was delivered; her Apgar scores were 8 and 9 at 1 and $5 \mathrm{~min}$, respectively. Abnormal bleeding vessels were noted around the uterine incision during the operation. The estimated blood loss was about $2,000 \mathrm{ml}$ and two units of packed red blood cells were required. Two hours after surgery the $\mathrm{Hb}$ count was $8.4 \mathrm{~g} / \mathrm{dl}$ and coagulation profile was normal. The rest of patient's postoperative course was unremarkable. To prevent thromboembolic disease, low-dose heparin (6,000 U/day) injection was administered for three postoperative days. The patient was discharged with her baby in good health.

A planned anesthesia for a KTS patient is challenging and with due emphasis on airway, hemodynamics, and neuraxial vascular malformation bleeding can result in a favorable outcome. Airway management in such patients may be difficult owing to the soft tissue hypertrophy characteristic of pregnancy and possible underestimated hemangiomas typical of KTS. Hemorrhagic complications may arise as a result of pelvic varicosities being injured by surgery [4]. A whole body MRI is recommended before performing a cesarean section to prevent hemorrhage complications and the anesthesiologist should pay attention to detect abnormal vessels of bronchial tube and lumbar spine [5]. In this case, an MRI was scheduled, but not executed yet. An MRI of the lumbar spine of six years before was negative for abnormal vessels around the spinal cord. Moreover, considering that an accurate examination of the spine and ultrasound scan excluded detectable central nervous system hemangiomas, we decided for a spinal anesthesia. Two more issues have influenced the choice of neuraxial technique: preoperative fasting recommendations were not respected because of urgency and the Mallampati class 3 airway revealed a probable difficult airway management. Given these concerns and a normal coagulation profile, a spinal anesthesia was executed assuming that a potential risk of difficult airway management and pulmonary aspiration were greater than possible complications correlated to the less probable presence of abnormal vessels near the spine. Another crucial point was the vasodilatation of the right leg varicosities after spinal anesthesia and its potential impact on hemodynamics, which could result in decreased venous return. The lifting of the leg by approximately thirty degrees increased venous return releasing the amount of blood eventually seized in the varicosities. A significant intraoperative blood loss should be expected and at least one unit of packed red blood cells should be readily available to be transfused as has been done in this case. The optimal management of a non-urgent scenario is to perform a neuraxial blockade only after having ascertained a negative spine MRI finding. In case of urgent cesarean section, the risks and benefits of each anesthesia technique must be compared.

\section{ORCID}

Gian Luigi Gonnella, https://orcid.org/0000-0002-7115-4408

Marco Scorzoni, https://orcid.org/0000-0002-3740-299X

Stefano Catarci, https://orcid.org/0000-0002-3466-6527

Bruno Antonio Zanfini, https://orcid.org/0000-0003-1312-5382

Gaetano Draisci, https://orcid.org/0000-0003-0148-5073

\section{References}

1. Samimi M, Lorette G. Klippel-Trenaunay syndrome. Presse Med 2010; 39: 487-94.

2. Gaiser RR, Cheek TG, Gutsche BB. Major conduction anesthesia in a patient with Klippel-Trenaunay Syndrome. J Clin Anesth 1995; 7: 316-9.

3. Sivaprakasam MJ, Dolak JA. Anesthetic and obstetric considerations in a parturient with Klippel-Trenaunay syndrome. Can J Anaesth 2006; 53: 487-91.

4. Hulsmans R. Complications of the pelvic involvement of Klippel-Trenaunay syndrome. Phlebologie 1991; 44: 404-6.

5. Tanaka R, Fujita Y, Ishibashi Hiasa K, Yumoto Y, Hidaka N, Fukushima K, et al. Successful management of pregnancy complicated by Klippel-Trenaunay syndrome using MR angiography-based evaluation. Case Rep Obstet Gynecol 2011; $2011: 723467$. 